\title{
DEVELOPMENT OF NIRS METHOD FOR ENERGETIC VALUE OF HIGH FIBER CANE CLONES
}

\author{
F DavrieuX ${ }^{1}$, C. Roussel ${ }^{2}, \mathrm{M} \mathrm{Mellin}^{2}$, R Cinier ${ }^{3}$, C Poser $^{4}$ \\ ${ }^{1}$ CIRAD, 7 chemin de I'IRAT, 97410 Saint-Pierre, La Réunion, France. \\ ${ }^{2}$ eRcane, 29 Rue d'Emmerez de Charmoy, 97494 Sainte Clotilde, La Réunion, France. \\ ${ }^{3}$ BRUKER OPTICS, 4 allée Hendrik Lorentz, Champs sur Marne 77447 Marne La Vallée, France. 4 CIRAD, \\ Station de la Bretagne, 97408 Saint-Denis Messagerie, Cedex 9, La Réunion France.
}

Corresponding author: davrieux@cirad.fr

Sypecar is a project carried out by Cirad which aims at studying high fiber cane for energetic valorization. One component of this project deals with fast and easy assessment of raw material using a semi-automatic Near Infra-Red Spectroscopy (NIRS) device (CPS instrument from Bruker based on Matrix F) dedicated to fresh cane analysis. Wet chemistry characterization consists in shredding stalk cane sample, pressing the resulting pulp in a hydraulic press and collecting plug and juice obtained. Then plug is weighed for determination of fibre content (fibre\% cane) while juice is filtered for determination of dry content (known as brix) and sugar content (known as pol). Those information were collected for high fiber cane and were completed by moisture content (MC) and Net Calorific Value (NCV). A calibration per component was developed. They were based on around 330 samples dataset from heterogeneous samples of the project Sypecar, including different varieties, two locations and varying ages. The predictions exhibited reliable results, since the coefficients of determination (R2) of regressions between predicted and observed values exceeded 0.9 for all traits and Ratio Performance to Deviation (RPD=Standard deviation/standard deviation of residuals) were higher than 4 . The performances for the two constituents of interest were promising: The fiber calibration SEP was $1.22 \%$, with a $\mathrm{R}^{2}$ equal to 0,96 and the NCV SEP was $147 \mathrm{kj} / \mathrm{kg}$, NCV minimum and maximum values were respectively 188 and $5022 \mathrm{Kj} / \mathrm{Kg}$, with a SD equal to $1209 \mathrm{Kj} / \mathrm{Kg}$.

KEYWORDS: Fiber cane, Net calorific value, FT-NIR Cane presentation system.

\section{INTRODUCTION}

In Reunion Island, 38\% of electricity is produced from renewable energies, including $10 \%$ from bagasse (fibrous matter remaining after sugarcane crushing). Policy makers wish to increase the part of renewable energies up to $50 \%$ in 2020 by developing different types of sustainable energy including biomass. SYPECAR project aims to determine potential production of energy cane in Reunion conditions. The starting assumption of the project is that the energy is generated by combustion of cane grown on marginal lands. The analytic part of the project aims to determine the cane properties and in particular the energetic potential of cane biomass (kJ/kg using NIRS on whole fresh cane.

Energy cane is not a specific phenotype and conversely the name "energy cane" gathers very diverse phenotypes from "high sugar-low fiber" to "low sugar-high fiber"1. Consequently, composition has to be determined: moisture, fiber, dry matter of juice, sugar and ash content and, in the end, the energetic potential of cane is evaluated by Net Calorific Value. The Net Calorific Value (NCV) represents the theoretical amount of recoverable energy held inside a unit mass of cane. Its direct measurement is long, difficult and expensive. Thus NCV is assessed by indirect methods using moisture content, dry content of juice and ash content.

The characterization of energy cane is mutli-analysis. Thanks to NIRS technology, this chracterization on whole fresh energy cane could be done in few operations : shredding cane, mixing and NIR measurement. That is why a NIRS device attached whith a semi-automatic cane presentation system were set up.

\section{MATERIALS AND METHODS}

The present study has been done on the full database including 476 NIRS spectra collected in 2014 and 2015. The dataset was first cleaned of outlying or suspicious spectra to develop reliable prediction equations. The database was constituted of 21 varieties of sugar cane: 12 varieties from genetic widening of eRcane collec- 
tion, 6 multi- purpose cultivars from Barbados and 3 commercial varieties. The 21 varieties were cultivated on two different pedoclimatic sites: Bérive in the south part of the island (altitude $670 \mathrm{~m}$, andosol, pluvial zone and average temperature $18^{\circ} \mathrm{C}$ ) and La Mare in the north part (altitude $70 \mathrm{~m}$, ferralitic soil, irrigated zone and average temperature $24^{\circ} \mathrm{C}$ ). Sampling was done at different ages of the plant: 10, 12, 14 months at Bérive and 8, 10, 12, 14 and 15 months at la Mare. On average, 3 samples were done per varieties and age modalities for each site.

The day after harvest whole fresh sugar canes were analyzed for their NIR spectrum using the Cane Presentation System (CPS, fig. 1) from Bruker (Bruker Optik GmbH, Ettlingen, Germany). To do this, about 5 to $10 \mathrm{~kg}$ of whole fresh cane were shredded using a Dedini D2500 II (Dedini, Piracicaba, SP, Brazil), then the pulp was introduced in the CPS and analysed. CPS system is an automatic start/stop system with a conveyor belt, based on a Bruker Matrix FTNIR, the wavelength range is 4000 to $12000 \mathrm{~cm}^{-1}$, the spectral resolution was $32 \mathrm{~cm}^{-1}$. Immediatly after NIR analysis, the pulp was analysed in the laboratory for the determination of water content (WC) 2, Dry content of juice (DCJ) and sugar content of juice (SCJ) (Method ICUMSA GS 7-31 2013), Fiber content (FC) based on Berding's formula ${ }^{3}$ and Net Calorific Value (NCV) using Wienese formula ${ }^{4}$. Statistical analyses and calibration development were performed using Opus 7.0 software (Bruker Optik GmbH, Ettlingen, Germany) and XLstat software (Addinsoft, Paris, France).
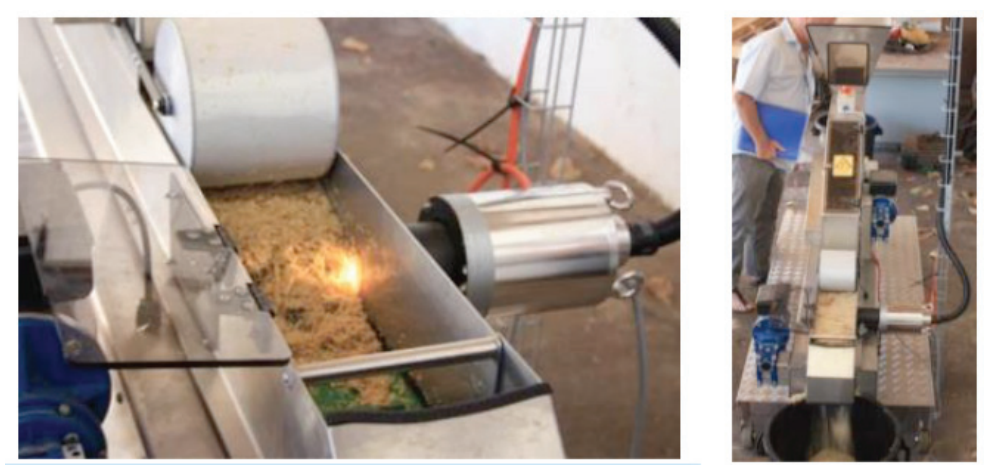

Figure 1: Cane Presentation System (CPS, Bruker)

The descriptive statistics of the constituents analyzed are reported in table1. The experimental design with a large panel of varieties and highly contrasting growing conditions ensured a wide range for each constituent of interest. So, water content ranged from $57,4 \%$ to $85,3 \%$ and SCJ from $0,01 \%$ to $16,8 \%$, that is to say from fiber cane to sugar cane. The result of this was that NCV values were representative of the variability with NCV values ranged from $469 \mathrm{Kj} / \mathrm{Kg}$ to more than $5000 \mathrm{Kj} / \mathrm{Kg}$.

Table 1. Descriptive statistics of the different constituents analyzed in the laboratory

\begin{tabular}{c|ccccc} 
Constituent & N & Minimum & Maximum & Mean & SD \\
\hline Dry content of Juice (\%) & 471 & 3.44 & 15.42 & 7.29 & 2.60 \\
Fiber content (\%/DM) & 471 & 11.19 & 31.10 & 21.10 & 4.69 \\
Water content (\%) & 473 & 57.44 & 85.30 & 71.89 & 6.50 \\
NCV (Kj/Kg) & 157 & 469 & 5315 & 2973 & 1259 \\
Sugar content of juice (\%) & 409 & 0.01 & 16.85 & 5.57 & 3.85
\end{tabular}

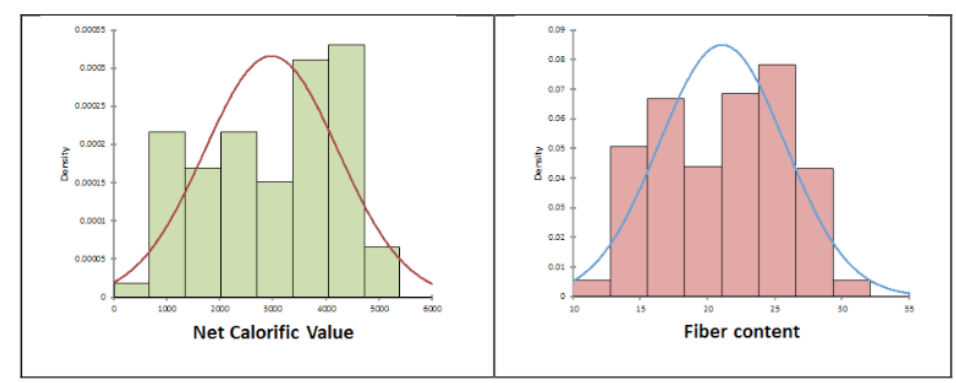

Figure 2: Histograms of NCV and Fiber content values. 
The distributions of the values for each parameter were Gaussian; however FC and NCV distribution were slightly bimodal due to the varieties and site effects (fig. 2).

The raw near infrared spectra were meanly characterized by the water absorbance bands (combination and harmonic bands), the second derivative spectrum of $\log (1 / R)$ highlighted absorption bands around $1469 \mathrm{~nm}(\mathrm{~N}$ $\mathrm{H}$, stretching) and $2406 \mathrm{~nm}(\mathrm{C}-\mathrm{H}$ stretching and $\mathrm{C}=\mathrm{O}$ combination bands). A principal component analysis was done on second derivative of the whole spectrum corrected for light scattering (SNVD correction). The scatter plot of samples scores for the two first PCs clearly showed two groups corresponding to the two sites (fig. 3). No sample was considerate as outlier according to Hotelling T2 distances.

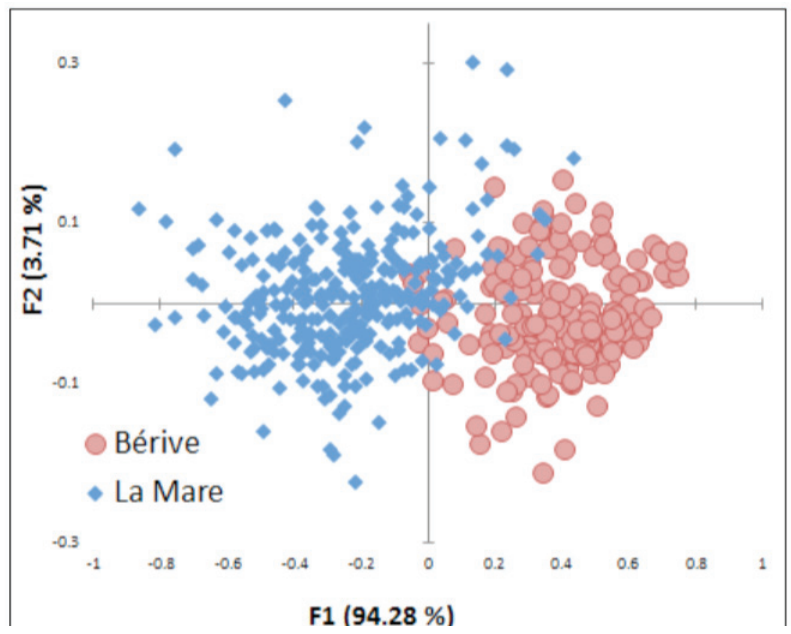

Figure 3: Scatter plots of samples scores for the two first PCs of PCA done on second derivative of NIR spectra.

This repartition was meanly due to water content and Fiber content which were significantly different for each site. The loadings associated to PC1 and PC2 clearly demonstrated it (data not shown) with high coefficients associated to water absorption bands.

The calibrations were developing using two sets of samples: calibration and validation. The validation set was constituted by random selection of 130 samples with the constraint that the percentage of samples per age and site modalities was similar to the whole data set one. The remaining 346 samples were used for calibration. Calibration parameters were optimized for the number of PLS terms, wavelength segment and spectrum pretreatments. The best combination of those parameters was chosen according to the optimum SECV and $R^{2}$ values. The calibrations parameters are reported in table 2. Calibrations were done using PLS regression.

Table 2. Calibration parameters.

\begin{tabular}{cccccccccc}
\hline Constituent & N & Mean & SD & Range & SEC & $\mathbf{R}^{\mathbf{2}}$ & SECV & Terms & t outliers \\
\hline DCJ & 323 & 7.02 & 2.54 & $3.44 \_15.42$ & 0.49 & 0.96 & 0.56 & 5 & 18 \\
FC & 323 & 20.59 & 4.67 & $11.62-29.49$ & 0.94 & 0.96 & 1.02 & 7 & 18 \\
WC & 327 & 72.50 & 6.51 & $57.44-85.30$ & 0.56 & 0.99 & 0.65 & 7 & 16 \\
SCJ & 286 & 5.14 & 3.76 & $0.01-16.85$ & 0.52 & 0.98 & 0.62 & 8 & 6 \\
NCV & 109 & 2802 & 1251 & $469-4864$ & 100 & 0.99 & 168 & 6 & 4 \\
\hline
\end{tabular}

Terms: number of PLS terms, $t$ outliers: number of samples removed of the calibration set according to $t$ test

Table 3. Validation parameters.

\begin{tabular}{cccccccccc}
\hline Constituent & N & Mean & SD & SEP & Bias & R $^{2}$ & slope & SEL & RPD \\
\hline DCJ & 127 & 7.54 & 2.45 & 0.56 & 0.06 & 0.952 & 1.007 & 0.07 & 4.4 \\
FC & 126 & 21.68 & 4.29 & 1.22 & -0.01 & 0.891 & 0.999 & 0.37 & 3.5 \\
WC & 127 & 70.85 & 6.21 & 0.76 & 0.00 & 0.985 & 0.989 & 0.30 & 8.1 \\
SCJ & 116 & 6.46 & 4.00 & 0.65 & -0.02 & 0.974 & 0.974 & 0.12 & 6.2 \\
NCV & 44 & 3309 & 1257 & 147 & -10.26 & 0.985 & 0.989 & 111 & 8.6 \\
\hline
\end{tabular}

RPD: SD/SEP, SEL: standard error of laboratory 
The standard errors of prediction (SEP) were estimated by predicting the validation set (table 3). The SEP are 3 to 8 times higher than the SEL depending of the constituent, however the NIR accuracy is very satisfactory and allows a fine varietal selection and a monitoring of the energy potential. The RPD Values observed are much higher than 3 , in particular for the determination of water content and NCV.

The scatter plots of the predicted values versus the laboratory values for both data sets : calibration and validation confirm the potential of NIR to analyse whole fresh sugar cane, the predictions are close to actual values for the whole range of values. This was especially relevant for WC and dry content of juice (figure 4), the prediction of these two parameters will be an efficient help for energy and selection programs. By calculation NCV is highly correlated to water content, the direct prediction of the calculated values was efficient, but in details the b coefficients of the NCV equation were highly noisly (data not shown) while WC b coefficients were sharp and well defined according to water absorption bands. However, an accurate prediction of WC allows an accurate estimation of NCV. The calibration for fiber content was less performant, however this calibration associated to the Dry content of juice and sugar content of juice equations will allow a high throughput screening of sugar cane varieties.
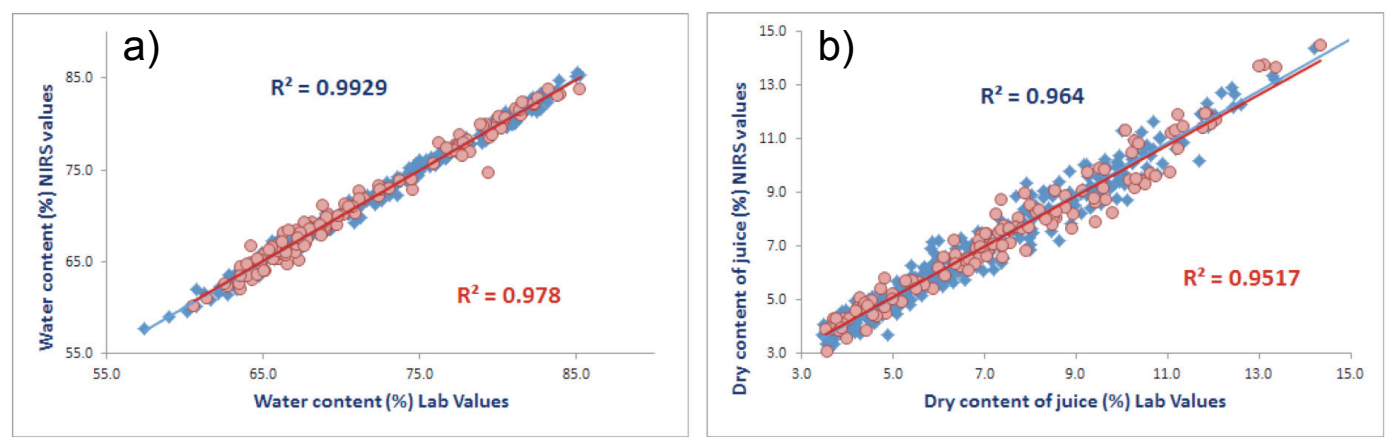

Figure 4. Scatter plots of the predicted values versus the laboratory values for WC (A) and DCJ (B). Calibration and validation sets.

\section{CONCLUSION}

The large genetic diversity and the highly different growing sites insure a well representative spectral data base, from fiber cane to sugar cane. The at line CPS-Matrix Bruker system demonstrated its efficiency and easy to handle for measurement of whole fresh cane. The models developed for the different parameters of sugar cane qualification presented high level of performances and will be applied as routine analysis as support of breeding and energy programs.

Regarding the time require to perform reference analysis, even for WC, the level of accuracy reached using the CPS, will improve the efficiency of the selection program. The NIR analysis of fresh cane for WC, fiber and NCV will greatly help the developpement of energy programs based on a selection of canes with high NCV potential. The calibration for water content determination was highly efficient (RPD=8.1, SEP=0.77\%) with a wide range ( $57 \%$ to $85 \%)$, the next step will be to adapt this calibration for monitoring cut canes during sun drying in the fields in order to catch the optimum WC corresponding to NCV optimum.

\section{Acknowledgments}

We thank the EU program FEADER and CIRAD for their funding support.

\section{References}

1. Matsuoka S. ; Kennedy A. J. ; dos Santos E. G. D. ; Tomazela A. L. ; Rubio L. C. S. ; Energy Cane: Its Concept, Development, Characteristics, and Prospects, 2014, Advances in Botany, Volume 2014, Article ID 597275. 2. Smit M.A. ; Martiné J.F. ; Kingston G. ; Van Den Berg M. ; Data collection standards for sugarcane growth monitoring, 2009, Issct Agronomy Workshop, 24-29 May 2009, Brazil

3. Berding N.; Pollock J.S.; Screening clones for fibre content with an hydraulic press, 1982, International Society of Sugar Cane Technologists Sugarcane Breeders' Newsletter 44, 43-48.

4. Wienese A. Boilers, boiler fuel and boiler efficiency, 2001, Proceedings of the South African Sugar Technologists Association, 75, 275-281. 